\title{
Dark Matter Detection with Bound Nuclear Targets: The Poisson Phonon Tail
}

\author{
Yonatan Kahn $\odot^{1,2, *}$ Gordan Krnjaic, ${ }^{3,4, \dagger}$ and Bashi Mandava $\oplus^{1, \$}$ \\ ${ }^{1}$ Department of Physics, University of Illinois at Urbana-Champaign, Urbana, Illinois 61801, USA \\ ${ }^{2}$ Illinois Center for Advanced Studies of the Universe, University of Illinois at Urbana-Champaign, \\ Urbana, Illinois 61801, USA \\ ${ }^{3}$ Fermi National Accelerator Laboratory, Batavia, Illinois 60510, USA \\ ${ }^{4}$ Kavli Institute for Cosmological Physics, University of Chicago, Chicago, Illinois 60637, USA
}

(Received 25 November 2020; revised 13 May 2021; accepted 26 July 2021; published 20 August 2021)

\begin{abstract}
Dark matter (DM) scattering with nuclei in solid-state systems may produce elastic nuclear recoil at high energies and single-phonon excitation at low energies. When the DM momentum is comparable to the momentum spread of nuclei bound in a lattice, $q_{0}=\sqrt{2 m_{N} \omega_{0}}$ where $m_{N}$ is the mass of the nucleus and $\omega_{0}$ is the optical phonon energy, an intermediate scattering regime characterized by multiphonon excitations emerges. We study a greatly simplified model of a single nucleus in a harmonic potential and show that, while the mean energy deposited for a given momentum transfer $q$ is equal to the elastic value $q^{2} /\left(2 m_{N}\right)$, the phonon occupation number follows a Poisson distribution and thus the energy spread is $\Delta E=q \sqrt{\omega_{0} /\left(2 m_{N}\right)}$. This observation suggests that low-threshold calorimetric detectors may have significantly increased sensitivity to sub-GeV DM compared to the expectation from elastic scattering, even when the energy threshold is above the single-phonon energy, by exploiting the tail of the Poisson distribution for phonons above the elastic energy. We use a simple model of electronic excitations to argue that this multiphonon signal will also accompany ionization signals induced from DM-electron scattering or the Migdal effect. In well-motivated models where DM couples to a heavy, kinetically mixed dark photon, we show that these signals can probe experimental milestones for cosmological DM production via thermal freeze-out, including the thermal target for Majorana fermion DM.
\end{abstract}

DOI: 10.1103/PhysRevLett.127.081804

The strategy of detecting dark matter (DM) through the energy imparted to a nucleus during a scattering process dates back to the 1980s [1,2] and is realized in leading contemporary experiments [3,4]. Designed for GeV-TeV scale DM, these experiments rapidly lose sensitivity to lighter DM. For elastic DM scattering off free nuclei, the energy transfer is inefficient if the DM mass $m_{\chi}$ is much below the nucleus mass $m_{N}$ : for $m_{\chi}=100 \mathrm{MeV}$, the typical momentum transfer is $q \sim m_{\chi} v \sim 150 \mathrm{keV}$, and the recoiling nucleus has energy $q^{2} /\left(2 m_{N}\right) \sim 0.1 \mathrm{eV}$, well below the thresholds of typical noble liquid detectors.

Recently, a vast landscape of plausible lighter DM models (see [5] and references therein) has necessitated revisiting the assumptions underlying nuclear scattering rate calculations. As emphasized in recent work [6,7], a nucleus in a lattice is not a free particle, and therefore elastic scattering does not necessarily occur in solid-state

Published by the American Physical Society under the terms of the Creative Commons Attribution 4.0 International license. Further distribution of this work must maintain attribution to the author(s) and the published article's title, journal citation, and DOI. Funded by SCOAP ${ }^{3}$. detectors. Indeed, an individual nucleus in a solid is subject to a restoring potential from its neighboring atoms

$$
V(r) \approx \frac{1}{2} m_{N} \omega_{0}^{2} r^{2},
$$

where $\omega_{0} \sim 60 \mathrm{meV}$ is a typical optical phonon energy for silicon and $r$ is the distance from the target atom. This potential acts only over interatomic distances $a \sim(\mathrm{keV})^{-1}$, so the displacement energy required to remove a nucleus from its lattice site is $E_{d} \sim \frac{1}{2} m_{N} \omega_{0}^{2} a^{2} \sim \mathcal{O}(10 \mathrm{eV})$. Only for recoil energies $E_{R}$ well above $E_{d}$ may the final-state nucleus be well approximated as a free particle, and the initial state is the ground state of a harmonic oscillator rather than a zero-momentum plane wave. This observation has previously been made in the context of single-phonon excitations in solids $[8,9]$, where DM with de Broglie wavelength $\lambda_{\mathrm{DB}}>a$ interacts primarily with phonons, quantized collective modes of many oscillating nuclei.

In this Letter, we study the intermediate regime $q \gg 1 / a$, such that the interaction is localized to a single lattice site, but with $E_{R} \lesssim E_{d}$ so that both the initial and final nuclear states belong to the harmonic oscillator spectrum. Our toy model of a solid-state detector treats each nucleus as a quantum harmonic oscillator subject 
to the potential in Eq. (1). The ground state momentum spread is

$$
q_{0}=\sqrt{2 m_{N} \omega_{0}},
$$

where $q_{0} \approx 56 \mathrm{keV}$ for silicon. We find that when a momentum transfer $q>q_{0}$ is kinematically allowed, the typical energy deposited is

$$
\bar{E}_{R}=\left(\frac{q}{q_{0}}\right)^{2} \omega_{0}=\frac{q^{2}}{2 m_{N}} .
$$

This is the energy expected from elastic scattering, but here it may be interpreted as the production of a multiphonon state with occupation number $n=\left(q / q_{0}\right)^{2}$.

We will show that in this greatly simplified model of a single oscillator, the distribution of phonon number is exactly Poissonian, so that unlike elastic scattering where only a single energy $E_{R}$ is allowed for a given $q$, there is a spread $\Delta n=q / q_{0}$ corresponding to an energy spread

$$
\Delta E_{R}=\left(\frac{q}{q_{0}}\right) \omega_{0}=q \sqrt{\frac{\omega_{0}}{2 m_{N}}},
$$

which extends the reach of solid-state nuclear recoil detectors sensitive to the total energy deposit from sub$\mathrm{GeV}$ DM compared to previous analyses which assumed the kinematics of elastic scattering. We verify that our results reduce to the previously calculated single-phonon rate when $q \ll q_{0}$-offering a pleasing interpretation of the single-phonon rate as an upward Poisson fluctuation when the elastic energy is well below the phonon energy-and converge exactly on the elastic recoil spectrum when $q \gg q_{0}$. Our work complements Refs. [6,7] by explicitly showing the transition between the single-phonon and elastic regimes in a simple model (see also [10] which considers production of multiple acoustic phonons for $q \ll q_{0}$ ). We will show that when the DM-nucleus interaction is mediated by a heavy dark photon, the full singleand multiphonon spectrum can probe a wide range of thermal relic targets.

Furthermore, we argue that when a nuclear recoil induces secondary ionization as in the Migdal effect [11-21] (Fig. 1), the electron spectrum factorizes from the phonon spectrum except at the very largest ionization energies. This result was previously known for isolated atoms [15], but we show that it persists in a simple model of a bound nucleus. Similarly, we also find that DM-electron scattering yields secondary phonon excitations. Therefore, the multiphonon spectrum we compute should be an irreducible component of an ionization signal in solid-state detectors, and due to the Poisson tail it may lie above the threshold of next-generation calorimetric detectors which would help distinguish a signal from other nonparticle backgrounds that create charge pairs without phonons. Finally, our results suggest a qualitatively different figure of merit for detector materials to detect sub-GeV DM-nuclear

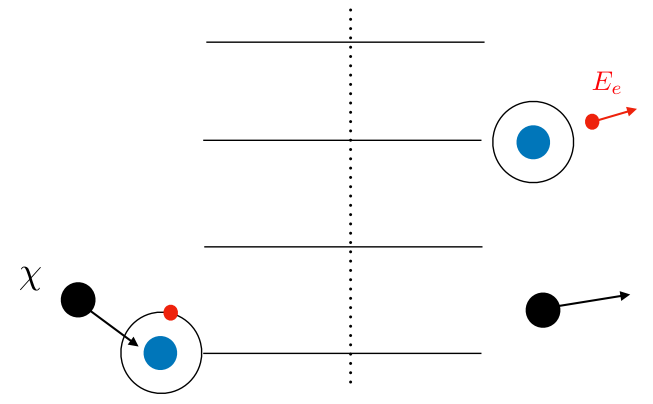

FIG. 1. Schematic cartoon of DM $\chi$ upscattering a bound nucleus in a harmonic oscillator ground state (left) into an excited state at level $n$ and possible additional ionization energy $E_{e}$ from the Migdal effect (right).

scattering: large phonon energies maximize the Poisson fluctuations when the elastic energy deposit is close to threshold.

Harmonic oscillator model.-We consider a single nucleus of mass $m_{N}$ subject to a three-dimensional isotropic harmonic oscillator potential:

$$
\hat{H}=\frac{\hat{\mathbf{p}}_{N}^{2}}{2 m_{N}}+\frac{m_{N} \omega_{0}^{2}}{2} \hat{\mathbf{r}}_{N}^{2} .
$$

The energy eigenstates are $\left|n_{x}, n_{y}, n_{z}\right\rangle$ with energies $E_{n}=\left(n+\frac{1}{2}\right) \omega_{0}$, where $n=n_{x}+n_{y}+n_{z}$. Following [22], we write the total DM scattering rate for a detector with $N_{T}$ nuclear targets as

$$
R=N_{T} \frac{\rho_{\chi}}{m_{\chi}} \frac{Z^{2} \bar{\sigma}_{p}}{8 \pi \mu_{\chi p}^{2}} \int \frac{d^{3} \mathbf{q}}{q} \sum_{n}|f(n, \mathbf{q})|^{2} \eta\left(v_{\min }^{(n)}\right),
$$

where $\rho_{\chi} / m_{\chi}=0.3 \mathrm{~cm}^{-3}\left(\mathrm{GeV} / m_{\chi}\right)$ is the local DM number density [23], $\mu_{\chi p}$ is the DM-proton reduced mass, $Z$ is the atomic number of the target, and $\bar{\sigma}_{p}$ is a fiducial DMproton cross section. For concreteness we restrict our attention to a contact interaction between DM and protons, but our results are easily generalizable to other interactions. We do not include a nuclear form factor because the allowed momentum transfers for sub-GeV DM are smaller than the inverse nuclear size, $q \ll 1 / R_{0} \sim \mathcal{O}(10 \mathrm{MeV})$ where $R_{0}$ is the nuclear radius.

The dimensionless functions appearing in the rate integral are the inelastic form factor

$$
|f(n, \mathbf{q})|^{2}=\sum_{n_{x}+n_{y}+n_{z}=n}\left|\left\langle n_{x}, n_{y}, n_{z}\left|e^{i \mathbf{q} \cdot \hat{\mathbf{r}}_{N}}\right| 0\right\rangle\right|^{2},
$$

and the DM inverse mean speed

$$
\eta\left(v_{\min }^{(n)}\right)=\int \frac{d^{3} \mathbf{v}}{v} f_{\chi}(\mathbf{v}) \Theta\left(v-v_{\min }^{(n)}\right),
$$

where $f_{\chi}(\mathbf{v})$ is the DM velocity distribution, which we assume to be the isotropic standard halo model. Here 


$$
v_{\min }^{(n)}=\frac{n \omega_{0}}{q}+\frac{q}{2 m_{\chi}}
$$

is the minimum DM speed needed to excite the nucleus to harmonic oscillator level $n$.

We calculate the form factor analytically using both momentum-space wave functions and the harmonic oscillator algebra in the Supplemental Material [24]. Defining an angular average $|f(n, q)|^{2} \equiv(1 / 4 \pi) \int d \Omega_{\mathbf{q}}|f(n, \mathbf{q})|^{2}$, we find

$$
|f(n, q)|^{2}=\frac{1}{n !}\left(\frac{q}{q_{0}}\right)^{2 n} e^{-q^{2} / q_{0}^{2}} .
$$

While to our knowledge this result has not appeared before in the DM literature, it is known in the case of neutron scattering [25]. Performing the angular average in Eq. (6) and using Eq. (10) yields a differential spectrum

$$
\frac{d R}{d q}=\frac{N_{T} \rho_{\chi}}{m_{\chi}} \frac{Z^{2} \bar{\sigma}_{p}}{2 \mu_{\chi p}^{2}} \sum_{n} \frac{q}{n !}\left(\frac{q}{q_{0}}\right)^{2 n} e^{-q^{2} / q_{0}^{2}} \eta\left(v_{\min }^{(n)}\right) .
$$

The form factor in (10) is nothing but a Poisson distribution in $n$ with mean $\bar{n}=q^{2} / q_{0}^{2}=q^{2} /\left(2 m_{N} \omega_{0}\right)$. Therefore, the mean energy deposited in a DM-nuclear scattering is $\bar{n} \omega_{0}=q^{2} /\left(2 m_{N}\right)$, the elastic value. In the limit $q^{2} \gg q_{0}^{2}$, the Poisson distribution approaches a delta function $\delta(n-\bar{n})$; taking the continuum limit $n \rightarrow E_{R} / \omega_{0}$, $\sum_{n} \rightarrow \int d n$ in Eq. (6), and replacing $q d q=m_{N} d E_{R}$, we recover the usual elastic recoil spectrum

$$
\frac{d R}{d E_{R}} \approx N_{T} m_{N} \frac{\rho_{\chi}}{m_{\chi}} \frac{Z^{2} \bar{\sigma}_{p}}{2 \mu_{\chi p}^{2}} \eta\left(v_{\min }^{(\bar{n})}\right),
$$

with $v_{\min }^{(\bar{n})}=\sqrt{m_{N} E_{R} /\left(2 \mu_{\chi N}^{2}\right)}$ where $\mu_{\chi N}$ is the DM-nucleus reduced mass. This matching justifies the standard approximation of approximating the nucleus as a free particle, at least for $\omega_{0} \ll E_{R}<E_{d}$ such that the nucleus remains bound.

Consider now the opposite limit, $q^{2} \ll q_{0}^{2}$. From [6-9], the form factor for production of a single optical phonon is parametrically $\left(q / q_{0}\right)^{2} e^{-q^{2} / q_{0}^{2}}$, where in the context of condensed matter physics the exponential is known as the (zero-temperature) Debye-Waller factor. We interpret this as the Poisson probability for $n=1$ phonons when $\bar{n} \ll 1$. The most likely excitation above the ground state is $n=1$, with larger phonon numbers strongly suppressed by powers of $q^{2} / q_{0}^{2} \ll 1$.

The advantage of our model is that it seamlessly interpolates between the single-phonon regime $q^{2} \ll q_{0}^{2}$ and the elastic regime $q^{2} \gg q_{0}^{2}$. When $q^{2} \sim q_{0}^{2}$, as is the case for kinematics of 10-50 MeV DM in the standard halo model, the Poissonian fluctuations in phonon number become important. In Fig. 2 we show representative quantized spectra that illustrate this behavior. Indeed, for

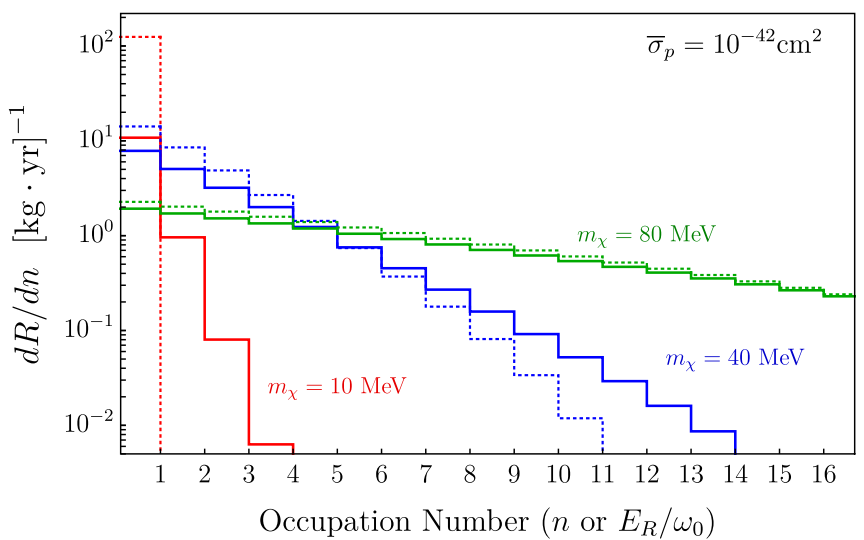

FIG. 2. Quantized event rate spectra from Eq. (6) using the angle-averaged Poisson form factor in Eq. (7) for various $m_{\chi}$. For comparison, the dotted histograms show the corresponding elastic recoil spectra quantized in units of $E_{R} / \omega_{0}$; the Poisson tail is clearly visible for smaller DM masses.

$10 \mathrm{MeV} \mathrm{DM}$, the rate above the single-phonon threshold is only a factor of 10 smaller than the single-phonon rate, allowing a detector with threshold above $\omega_{0}$ to be sensitive to this low-mass DM.

Nuclear scattering reach.-Here we estimate the reach of a next-generation calorimetric detector. For concreteness, we consider a DM-nucleus interaction mediated by a massive, kinetically mixed dark photon $A^{\prime}$ with Lagrangian

$$
\mathcal{L}_{\text {int }}=A_{\mu}^{\prime}\left(\epsilon e J_{\mathrm{EM}}^{\mu}+g_{D} J_{D}^{\mu}\right),
$$

where $\epsilon$ is the kinetic mixing, $g_{D}$ is the $A^{\prime}-\chi$ coupling constant, $J_{\mathrm{EM}}^{\mu}$ is the electromagnetic current, and $J_{D}^{\mu}$ is the DM current. In the contact limit $m_{A^{\prime}} \gg q$, for complex scalar $\chi$, the single-proton cross section is

$$
\bar{\sigma}_{p}=\frac{16 \pi \epsilon^{2} \alpha \alpha_{D} \mu_{\chi p}^{2}}{m_{A^{\prime}}^{4}}
$$

which enters into Eq. (6). For $m_{A^{\prime}}>m_{\chi}$, this model can realize thermal freeze-out via $\chi \chi^{*} \rightarrow f^{+} f^{-}$annihilation where $f$ is a charged fermion, and there is a one-to-one correspondence between $\bar{\sigma}_{p}$ and the early universe annihilation rate $[26,27]$.

Figure 3 shows how the Poisson phonon tail can enhance the DM signal yield for detectors with various energy thresholds, from $1 \mathrm{eV}$ down to the single-phonon energy $\omega_{0}$. Each solid curve represents a different threshold for a silicon crystal target. We only include transitions into bound oscillator final states $0 \rightarrow n$ with $n \omega_{0}<E_{d}$, so this simple model represents a lower bound on the total signal rate. The dashed curves show the would-be sensitivity of a low-threshold detector in the elastic regime, demonstrating that our result interpolates between the discrete phonon regime and elastic scattering, and that multiphonon production provides increased sensitivity at lower DM masses. 


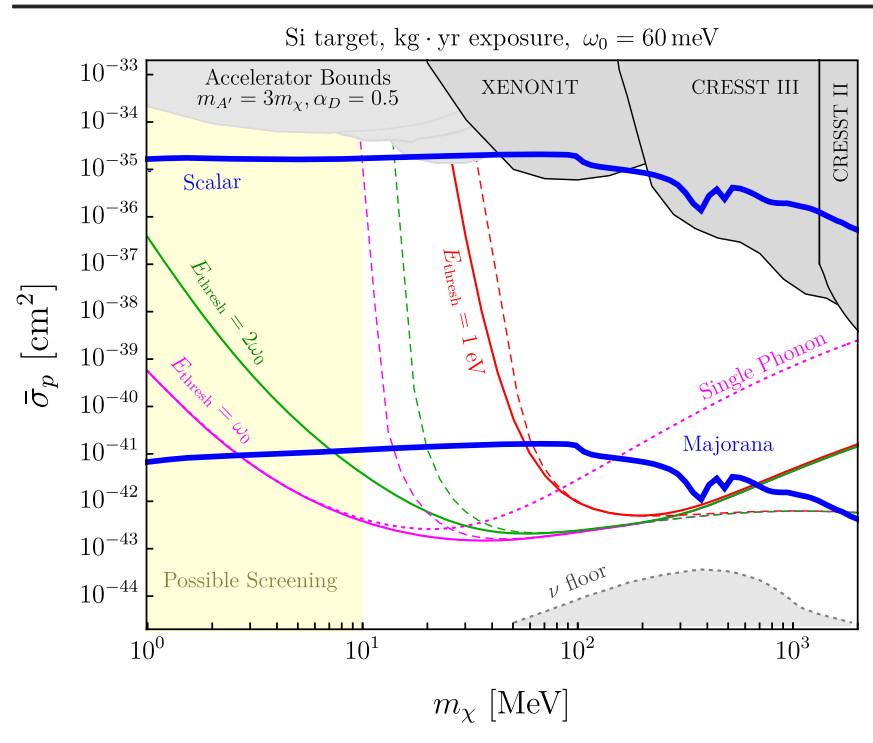

FIG. 3. Exclusion curves (three events, zero background) for various energy thresholds computed using Eq. (6) with $n \leq$ $E_{\text {thresh }} / \omega_{0}$ (solid), along with elastic scattering (long dashed) and single-phonon production (short dashed magenta). Constraints from accelerators [28-30], beam dumps [31-35], and direct detection [36-38] (converting from electron scattering to $\bar{\sigma}_{p}$ in the dark photon model where necessary) are shaded grey, along with the neutrino floor [5]. The yellow shaded region $m_{\chi}<$ $10 \mathrm{MeV}$ shows where screening may be important.

Note that, as in [7], the dashed elastic curves in this figure flatten towards higher masses reflecting the $\mu_{\chi p}$ dependence in Eq. (12); the inelastic phonon curves shift upwards as a greater fraction of DM can deposit energy above $E_{d}$ to displace the nucleus, which we do not consider in our model.

Since the $A^{\prime}$ couples universally to charge, we modify Eq. (6) to account for screening through an atomic form factor [39-41]:

$$
\left|F_{A}(q)\right|^{2}=\frac{\left(\lambda_{\mathrm{TF}}^{2} q^{2}\right)^{2}}{\left(1+\lambda_{\mathrm{TF}}^{2} q^{2}\right)^{2}},
$$

where $\lambda_{\mathrm{TF}} \approx\left(0.89 / Z^{1 / 3}\right) a_{0} \approx 0.37 a_{0}$ is the Thomas-Fermi screening length for silicon and $a_{0}$ is the Bohr radius. At low momentum $q \ll 1 / \lambda_{\mathrm{TF}},\left|F_{A}(q)\right|^{2} \rightarrow 0$, reflecting complete charge screening by the neutral atom. This is also a manifestation of the well-known fact that dark photons do not couple efficiently to optical phonons at low momentum in nonpolar crystals, since out-of-phase oscillations are suppressed compared to in-phase oscillations by powers of $q$. However, because $\lambda_{\mathrm{TF}} q_{0} \approx 5.2$ in silicon, $\left|F_{A}\left(q_{0}\right)\right|^{2}=0.93$, so this screening only affects the kinematic regime with $q \lesssim q_{0}$. In practice, screening slightly suppresses the single-phonon rate and the total rate below $m_{\chi} \simeq 10 \mathrm{MeV}$ (shaded yellow in Fig. 3) compared to a generic heavy mediator coupling only to protons. For larger DM masses, our single-phonon curve roughly reproduces the analysis of [7] for a heavy hadrophilic mediator, an important check on the validity of this simple model.

The blue curves in Fig. 3 correspond to thermal freezeout targets for complex scalar and Majorana fermion DM candidates coupled to $A^{\prime}$. The Majorana cross section is proportional to $\bar{\sigma}_{p}$ in Eq. (14) and further suppressed by $v^{2} \sim 10^{-6}$ (see [27] for details). Both models here feature $p$-wave DM annihilation and are, therefore, safe from cosmic microwave background bounds, which exclude freeze-out for $s$-wave candidates with $m_{\chi} \lesssim 10 \mathrm{GeV}$ [42]. It is notable that calorimeters with thresholds in the few-phonon range may soon begin to explore the Majorana thermal target.

Migdal effect and electron scattering.-Nearly a century ago Migdal observed that a sudden impulse delivered to the nucleus could result in electronic transitions in atoms [11]. This improves the possibility of detecting nuclear recoil events through secondary ionization even if the nuclear recoil is below threshold, because the ionization energy may greatly exceed the (possibly quenched) elastic energy from nuclear recoil. However, as noted in [18,21], strictly speaking the Migdal effect only applies to isolated atoms, where the energy eigenstates are plane waves and thus one may boost to the frame of the recoiling nucleus. Recently, Ref. [21] provided a rigorous derivation of the Migdal effect in semiconductors, including many-body electron states such as the plasmon [43,44] and improving on previous calculations which used the free-nucleus approximation $[15,19]$.

Here, in the spirit of our nuclear scattering analysis, we add to our simple model a potential $V_{e}$ acting on a single electron:

$$
\hat{H}=\frac{\hat{\mathbf{p}}_{N}^{2}}{2 m_{N}}+\frac{\hat{\mathbf{p}}_{e}^{2}}{2 m_{e}}+\frac{m_{N} \omega_{0}^{2}}{2} \hat{\mathbf{r}}_{N}^{2}+V_{e}\left(\hat{\mathbf{r}}_{N}-\hat{\mathbf{r}}_{e}\right) .
$$

Transforming to relative and center-of-mass coordinates, $\hat{\mathbf{r}} \equiv \hat{\mathbf{r}}_{N}-\hat{\mathbf{r}}_{e}, \hat{\mathbf{R}}=\left(m_{N} \hat{\mathbf{r}}_{N}+m_{e} \hat{\mathbf{r}}_{e}\right) /\left(m_{N}+m_{e}\right)$, the Hamiltonian becomes separable up to small perturbations (see Supplemental Material), and the eigenstates are $|\Psi\rangle=\left|\vec{n} ; \psi_{e}\right\rangle$, where the first label is the harmonic oscillator state and $\psi_{e}(\mathbf{r})$ is the electronic wave function. The transition amplitudes from Eq. (7) now factorize [45] into oscillator and ionization terms

$$
\left\langle\Psi^{\prime}\left|e^{i \mathbf{q} \cdot \hat{\mathbf{r}}_{N}}\right| \Psi\right\rangle \simeq\left\langle\vec{n}\left|e^{i \mathbf{q} \cdot \hat{\mathbf{R}}}\right| 0\right\rangle\left\langle\psi_{e}^{\prime}\left|e^{i \mathbf{q}_{e} \cdot \hat{\mathbf{r}}}\right| \psi_{e}\right\rangle
$$

where $\mathbf{q}_{e} \equiv\left(m_{e} / m_{N}\right) \mathbf{q}$ [15]. If the ionization matrix element depends only on the magnitude $\left|\mathbf{q}_{e}\right|$, the differential scattering rate factorizes

$$
\frac{d R}{d q d E_{e}}=\frac{N_{T} \rho_{\chi}}{m_{\chi}} \frac{Z^{2} \bar{\sigma}_{p}}{2 \mu_{\chi p}^{2}} \sum_{n} q|f(n, q)|^{2} \sum_{f} \frac{d\left|Z_{f}\right|^{2}}{d E_{e}} \eta\left(v_{\min }^{(n, e)}\right),
$$


where $f(n, q)$ is the Poisson distribution in Eq. (10) and

$$
\left|Z_{f}\right|^{2} \equiv\left|\left\langle\psi_{e}^{\prime}\left|e^{i \mathbf{q}_{e} \cdot \hat{\mathbf{r}}}\right| \psi_{e}\right\rangle\right|^{2}
$$

is the ionization probability as in [15]; if $Z_{f}$ does not factorize, the integration is a convolution of the ionization and oscillator form factors and may deviate from the Poisson form (see Supplemental Material).

In this model, the electronic spectrum is unmodified to leading order in $m_{e} / m_{N}$ compared to the free-nucleus picture, and the only changes come in replacing the initial and final nuclear states with harmonic oscillator states, the matrix elements of which we have already calculated. Equation (17) implies the only coupling between the electronic and nuclear excitation energies in Eq. (18) comes from energy conservation, which after integrating over the DM velocity distribution modifies $v_{\min }^{(n)}$ in Eq. (9) by $n \omega_{0} \rightarrow n \omega_{0}+E_{e}$. As our earlier analysis has shown, the mean nuclear energy is the elastic energy, which is typically much less than the electronic excitation energy. Thus, the only effect of the harmonic oscillator spectrum is to truncate the electronic spectrum at large $E_{e}$ due to the Poisson tail, a conclusion which qualitatively agrees with Ref. [21].

On the other hand, for DM scattering directly off an electron $[22,46]$, the matrix element is proportional to $\left\langle\Psi^{\prime}\left|e^{i \mathbf{q} \cdot \hat{\mathbf{r}}_{e}}\right| \Psi\right\rangle$, which now involves $\mathbf{r}_{e}=\mathbf{R}-\left(\mu / m_{e}\right) \mathbf{r}$, where $\mu \equiv m_{e} m_{N} /\left(m_{e}+m_{N}\right) \approx m_{e}$. In the limit $m_{N} \gg m_{e}$, the above analysis yields

$$
\left\langle\Psi^{\prime}\left|e^{i \mathbf{q} \cdot \hat{\mathbf{r}}_{e}}\right| \Psi\right\rangle \simeq\left\langle\vec{n}\left|e^{i \mathbf{q} \cdot \hat{\mathbf{R}}}\right| 0\right\rangle\left\langle\psi_{e}^{\prime}\left|e^{-i \mathbf{q} \cdot \hat{\mathbf{r}}}\right| \psi_{e}\right\rangle
$$

where the electron matrix element, $\left\langle\psi_{e}^{\prime}\left|e^{-i \mathbf{q} \cdot \hat{\mathbf{r}}}\right| \psi_{e}\right\rangle$, is familiar from previous analyses of DM-electron scattering, but the nuclear matrix element is identical to that of the Migdal effect.

In this simplified model, our analysis of the nuclear scattering matrix element suggests that the Poisson tail may help push the nuclear recoil energies accompanying ionization signals above threshold. A detector capable of converting ionization energy to phonons, such as the low-threshold calorimetric detectors used by SuperCDMS [47-49] and EDELWEISS [50,51], could observe the Poisson phonon spectrum of nuclear recoil simultaneously with the electron-hole pairs created by ionization, which could help distinguish between true scattering events and low-momentum-transfer background processes such as charge leakage.

Conclusions. - We have presented a simple quantummechanical model which describes the multiphonon regime of DM-nuclear scattering in solid-state systems, interpolating between the elastic regime and single-phonon production when the energy deposit is less than the displacement energy $E_{d}$. Our key finding is that when the elastic energy $q^{2} / 2 m_{N}$ is close to the optical phonon energy $\omega_{0}$, there is an order-1 variance in the phonon yield, such that the sensitivity of low-threshold detectors to low-mass DM is stronger than previously expected from elastic kinematics alone. This observation suggests that detectors with an especially large optical phonon energy, such as diamond [52], may be able to take advantage of these Poisson fluctuations even when the detector threshold is somewhat above the single-phonon energy.

As an illustrative example, we have shown that plausible next-generation detectors with $\sim \mathrm{eV}$ scale thresholds sensitive to total energy deposit [53] can exploit this irreducible effect to greatly enhance their sensitivity to sub-GeV DM. Remarkably, the potential gains identified here could enable such detectors to probe the full thermal relic parameter space for Majorana DM candidates with mass between $10 \mathrm{MeV}$ and $1 \mathrm{GeV}$ freezing out via kinetically mixed dark photons, corresponding to parameter space far below current limits.

Finally, according to [25], a more realistic model would incorporate a nontrivial phonon density of states and anharmonicities which broaden the phonon spectrum, but we expect that the parametric scaling of our result would persist, at least for monoatomic crystals. Indeed, our results are qualitatively consistent with Ref. [21] where the full phonon density of states was included and the multiphonon response had a mean and width approximately consistent with a Poisson distribution for $q / q_{0} \gtrsim 2$. Crystals with more than one atom per unit cell, in particular polar materials where dark photons couple to optical phonons even at low $q$ [6-9], may exhibit similar behavior but deserve a dedicated analysis, especially because of their directional detection capabilities. We emphasize that the rates we have computed represent lower bounds on the total DM-nuclear signal rate. Indeed, DM heavier than about $10 \mathrm{MeV}$ has sufficient kinetic energy to displace a nucleus from its lattice site, and the fact that neither the initial nor the final states are free plane waves may allow for the possibility of inelastic scattering when the elastic rate below threshold is zero. This would give a spectrum of recoil events with $E_{R}>E_{d}$ additive to the one we consider here. We plan to investigate this possibility in future work.

We thank Daniel Baxter, Gordon Baym, Simon Knapen, Jonathan Kozaczuk, Noah Kurinsky, and Tongyan Lin for many enlightening discussions. The work of Y. K. is supported in part by US Department of Energy Grant No. DE-SC0015655. Fermilab is operated by the Fermi Research Alliance, LLC under Contract No. DE-AC0207CH11359 with the U.S. Department of Energy.

Note added.-The following Letter [21] complements this Letter with a study of the Migdal effect in realistic semiconductor materials and the associated multiphonon response. 
*yfkahn@illinois.edu

${ }^{\dagger}$ krnjaicg@fnal.gov

*mandava3@illinois.edu

[1] M. W. Goodman and E. Witten, Detectability of certain dark matter candidates, Phys. Rev. D 31, 3059 (1985).

[2] A. K. Drukier, K. Freese, and D. N. Spergel, Detecting cold dark matter candidates, Phys. Rev. D 33, 3495 (1986).

[3] E. Aprile et al. (XENON Collaboration), Projected WIMP sensitivity of the XENONnT dark matter experiment, J. Cosmol. Astropart. Phys. 11 (2020) 031.

[4] D. Akerib et al. (LZ Collaboration), The LUX-ZEPLIN (LZ) experiment, Nucl. Instrum. Methods Phys. Res., Sect. A 953, 163047 (2020).

[5] M. Battaglieri et al., US cosmic visions: New ideas in dark matter 2017: Community Report, 2017.

[6] T. Trickle, Z. Zhang, K. M. Zurek, K. Inzani, and S. Griffin, Multi-channel direct detection of light dark matter: Theoretical framework, J. High Energy Phys. 03 (2020) 036.

[7] S. M. Griffin, K. Inzani, T. Trickle, Z. Zhang, and K. M. Zurek, Multichannel direct detection of light dark matter: Target comparison, Phys. Rev. D 101, 055004 (2020).

[8] S. Knapen, T. Lin, M. Pyle, and K. M. Zurek, Detection of light dark matter with optical phonons in polar materials, Phys. Lett. B 785, 386 (2018).

[9] S. Griffin, S. Knapen, T. Lin, and K. M. Zurek, Directional detection of light dark matter with polar materials, Phys. Rev. D 98, 115034 (2018).

[10] B. Campbell-Deem, P. Cox, S. Knapen, T. Lin, and T. Melia, Multiphonon excitations from dark matter scattering in crystals, Phys. Rev. D 101, 036006 (2020); Erratum, Phys. Rev. D 102, 019904 (2020).

[11] A. Migdal, Ionizatsiya atomov pri yadernykh reaktsiyakh, Sov. Phys. JETP 9, 1163 (1939).

[12] J. D. Vergados and H. Ejiri, The role of ionization electrons in direct neutralino detection, Phys. Lett. B 606, 313 (2005).

[13] C. C. Moustakidis, J. D. Vergados, and H. Ejiri, Direct dark matter detection by observing electrons produced in neutralino-nucleus collisions, Nucl. Phys. B727, 406 (2005).

[14] R. Bernabei et al., On electromagnetic contributions in WIMP quests, Int. J. Mod. Phys. A 22, 3155 (2007).

[15] M. Ibe, W. Nakano, Y. Shoji, and K. Suzuki, Migdal effect in dark matter direct detection experiments, J. High Energy Phys. 03 (2018) 194.

[16] M. J. Dolan, F. Kahlhoefer, and C. McCabe, Directly Detecting Sub-GeV Dark Matter with Electrons from Nuclear Scattering, Phys. Rev. Lett. 121, 101801 (2018).

[17] N. F. Bell, J. B. Dent, J. L. Newstead, S. Sabharwale, and T. J. Weiler, The Migdal effect and photon Bremsstrahlung in effective field theories of dark matter direct detection and coherent elastic neutrino-nucleus scattering, Phys. Rev. D 101, 015012 (2020).

[18] D. Baxter, Y. Kahn, and G. Krnjaic, Electron ionization via dark matter-electron scattering and the Migdal effect, Phys. Rev. D 101, 076014 (2020).

[19] R. Essig, J. Pradler, M. Sholapurkar, and T.-T. Yu, Relation between the Migdal Effect and Dark Matter-Electron Scattering in Isolated Atoms and Semiconductors, Phys. Rev. Lett. 124, 021801 (2020).
[20] G. Grilli di Cortona, A. Messina, and S. Piacentini, Migdal effect and photon Bremsstrahlung: Improving the sensitivity to light dark matter of liquid argon experiments, J. High Energy Phys. 11 (2020) 034.

[21] S. Knapen, J. Kozaczuk, and T. Lin, following Letter, The Migdal Effect in Semiconductors, Phys. Rev. Lett. 127, 081805 (2021).

[22] R. Essig, M. Fernandez-Serra, J. Mardon, A. Soto, T. Volansky, and T.-T. Yu, Direct detection of sub-GeV dark matter with semiconductor targets, J. High Energy Phys. 05 (2016) 046.

[23] P. F. de Salas, Dark matter local density determination based on recent observations, J. Phys. Conf. Ser. 1468, 012020 (2020).

[24] See Supplemental Material at http://link.aps.org/ supplemental/10.1103/PhysRevLett.127.081804 for more details.

[25] H. Schober, An introduction to the theory of nuclear neutron scattering in condensed matter, J. Neutron Res. 17, 109 (2014).

[26] E. Izaguirre, G. Krnjaic, P. Schuster, and N. Toro, Analyzing the Discovery Potential for Light Dark Matter, Phys. Rev. Lett. 115, 251301 (2015).

[27] A. Berlin, N. Blinov, G. Krnjaic, P. Schuster, and N. Toro, Dark matter, millicharges, axion and scalar particles, gauge bosons, and other new physics with LDMX, Phys. Rev. D 99, 075001 (2019).

[28] E. Izaguirre, G. Krnjaic, P. Schuster, and N. Toro, New electron beam-dump experiments to search for $\mathrm{MeV}$ to fewGeV dark matter, Phys. Rev. D 88, 114015 (2013).

[29] R. Essig, J. Mardon, M. Papucci, T. Volansky, and Y.-M. Zhong, Constraining light dark matter with low-energy $e^{+} e^{-}$colliders, J. High Energy Phys. 11 (2013) 167.

[30] J. Lees et al. (BABAR Collaboration), Search for Invisible Decays of a Dark Photon Produced in $e^{+} e^{-}$Collisions at $B A B A R$, Phys. Rev. Lett. 119, 131804 (2017).

[31] P. deNiverville, M. Pospelov, and A. Ritz, Observing a light dark matter beam with neutrino experiments, Phys. Rev. D 84, 075020 (2011).

[32] D. Banerjee et al., Dark Matter Search in Missing Energy Events with NA64, Phys. Rev. Lett. 123, 121801 (2019).

[33] B. Batell, R. Essig, and Z. Surujon, Strong Constraints on Sub-GeV Dark Sectors from SLAC Beam Dump E137, Phys. Rev. Lett. 113, 171802 (2014).

[34] L. Marsicano, M. Battaglieri, M. Bondí, C. D. R. Carvajal, A. Celentano, M. De Napoli, R. De Vita, E. Nardi, M. Raggi, and P. Valente, Novel Way to Search for Light Dark Matter in Lepton Beam-Dump Experiments, Phys. Rev. Lett. 121, 041802 (2018).

[35] A. A. Aguilar-Arevalo, Miniboone-dm: A dark matter search in a proton beam dump, J. Phys. Conf. Ser. 1342, 012055 (2020).

[36] E. Aprile et al. (XENON Collaboration), Light Dark Matter Search with Ionization Signals in XENON1T, Phys. Rev. Lett. 123, 251801 (2019).

[37] A. Abdelhameed et al. (CRESST Collaboration), First results from the CRESST-III low-mass dark matter program, Phys. Rev. D 100, 102002 (2019). 
[38] A. Abdelhameed et al. (CRESST Collaboration), First results from the CRESST-III low-mass dark matter program, Phys. Rev. D 100, 102002 (2019).

[39] L. Schiff, Energy-angle distribution of thin target Bremsstrahlung, Phys. Rev. 83, 252 (1951).

[40] Y.-S. Tsai, Pair production and Bremsstrahlung of charged leptons, Rev. Mod. Phys. 46, 815 (1974); 49, 421(E) (1977).

[41] T. Emken, R. Essig, C. Kouvaris, and M. Sholapurkar, Direct detection of strongly interacting sub-GeV dark matter via electron recoils, J. Cosmol. Astropart. Phys. 09 (2019) 070 .

[42] N. Aghanim et al. (Planck Collaboration), Planck 2018 results. VI. Cosmological parameters, Astron. Astrophys. 641, A6 (2020).

[43] N. Kurinsky, D. Baxter, Y. Kahn, and G. Krnjaic, Dark matter interpretation of excesses in multiple direct detection experiments, Phys. Rev. D 102, 015017 (2020).

[44] J. Kozaczuk and T. Lin, Plasmon production from dark matter scattering, Phys. Rev. D 101, 123012 (2020).

[45] Although our toy model of phonons as harmonic oscillator states exhibits leading order factorization in Eq. (17), such behavior may not necessarily hold in a more sophisticated treatment of the phonon density of states [21]. However, a more detailed analysis of this point is beyond the scope of this work.

[46] R. Essig, J. Mardon, and T. Volansky, Direct detection of sub-GeV dark matter, Phys. Rev. D 85, 076007 (2012).
[47] N. Kurinsky, P. Brink, R. Partridge, B. Cabrera, and M. Pyle (SuperCDMS Collaboration), SuperCDMS SNOLAB lowmass detectors: Ultra-sensitive phonon calorimeters for a sub-GeV dark matter search, Proc. Sci. ICHEP2016 (2017) 1116 [arXiv:1611.04083].

[48] R. Agnese et al. (SuperCDMS Collaboration), First Dark Matter Constraints from a SuperCDMS Single-Charge Sensitive Detector, Phys. Rev. Lett. 121, 051301 (2018); Erratum, Phys. Rev. Lett. 122, 069901 (2019).

[49] I. Alkhatib et al. (SuperCDMS Collaboration), Light Dark Matter Search with a High-Resolution Athermal Phonon Detector Operated Above Ground, arXiv:2007.14289 [Phys. Rev. Lett. (to be published)].

[50] E. Armengaud, C. Augier, A. Benoît, A. Benoit, L. Bergé et al. (EDELWEISS Collaboration), Searching for low-mass dark matter particles with a massive Ge bolometer operated above-ground, Phys. Rev. D 99, 082003 (2019).

[51] Q. Arnaud, E. Armengaud, C. Augier, A. Benoît, L. Bergé et al. (EDELWEISS Collaboration), First Germanium-Based Constraints on Sub-MeV Dark Matter with the EDELWEISS Experiment, Phys. Rev. Lett. 125, 141301 (2020).

[52] N. A. Kurinsky, T. C. Yu, Y. Hochberg, and B. Cabrera, Diamond detectors for direct detection of sub-GeV dark matter, Phys. Rev. D 99, 123005 (2019).

[53] R. Ren et al., Design and characterization of a phononmediated cryogenic particle detector with an eV-scale threshold and $100 \mathrm{keV}$-scale dynamic range, arXiv:2012.12430 [Phys. Rev. D (to be published)]. 